\title{
$\angle S$ Research Square \\ Sulfur intermediates as new biogeochemical hubs in an aquatic model microbial ecosystem
}

Adrien Vigneron

Universite Laval https://orcid.org/0000-0003-3552-8369

Perrine Cruaud

Universite Laval

Alexander I. Culley

Universite Laval

Raoul-Marie Couture

Universite Laval

Connie Lovejoy ( $\sim$ Connie.Lovejoy@bio.ulaval.ca )

https://orcid.org/0000-0001-8027-2281

Warwick F. Vincent

Universite Laval

Research

Keywords: meromictic lake, anoxic basin, Arctic Ocean, sulfur cycling, Organic sulfur molecules, sulfur cycle intermediates, redox gradients, metagenomics

Posted Date: June 2nd, 2020

DOI: https://doi.org/10.21203/rs.3.rs-32029/v1

License: (c) (1) This work is licensed under a Creative Commons Attribution 4.0 International License.

Read Full License 


\section{Abstract}

\section{Background}

The sulfur cycle encompasses a series of complex aerobic and anaerobic transformations of Scontaining molecules, and plays a fundamental role in cellular and ecosystems level-processes, influencing biological carbon transfers and other biogeochemical cycles. Despite their importance, the microbial communities and metabolic pathways involved in these transformations remain poorly understood, notably for inorganic sulfur compounds of intermediate oxidation states (thiosulfate, tetrathionate, sulfite, polysulfides). Isolated and highly stratified, the extreme geochemical and environmental contexts of the meromictic ice-capped Lake A, in the Canadian High Arctic, provides an outstanding model ecosystem to resolve the distribution and metabolism of aquatic sulfur cycling microorganisms along redox and salinity gradients.

\section{Results}

Applying complementary molecular approaches, we identified sharply contrasting microbial communities and metabolic potentials among the distinct water layers of the Lake A, with homologies to diverse fresh, brackish and saline water microbiomes. Sulfur cycling genes were abundant at all depths, with oxidative processes in the oxic freshwater layers, reductive reactions in the anoxic and sulfidic bottom waters and genes for both transformations at the chemocline, and co-varied with bacterial abundance. Up to 154 different genomic bins with potential for sulfur transformation were recovered, revealing a panoply of taxonomically diverse microorganisms with complex metabolic pathways for biogeochemical sulfur reactions. Metabolism of sulfur cycle intermediates was widespread throughout the water column, cooccurring with sulfate reduction or sulfide oxidation pathways. The genomic bin composition suggested that in addition to chemical oxidation, these intermediate sulfur compounds were likely produced by the predominant sulfur chemo- and photo-oxidizers at the chemocline and by diverse microbial organic sulfur molecule degraders.

\section{Conclusions}

The Lake A microbial ecosystem provided an ideal opportunity to identify new features of the biogeochemical sulfur cycle. Our detailed metagenomic analyses across the broad physico-chemical gradients of this highly stratified lake extend the known diversity of microorganisms and metabolic pathways involved in sulfur transformations over a wide range of environmental conditions. The results identify the importance of sulfur cycle intermediates and organic sulfur molecules as major sources of electron donors and acceptors for aquatic and sedimentary microbial communities in association with the classical sulfur cycle.

\section{Background}


The sulfur cycle encompasses complex energetic processes where sulfur (S) ions and molecules in different oxidation states, from the most reduced (-2: sulfides, $\left.\mathrm{H}_{2} \mathrm{~S} / \mathrm{HS}^{-}\right)$to the most oxidized ( +6 : sulfate, $\mathrm{SO}_{4}{ }^{2-}$ ), are transformed through oxidation, reduction, disproportionation [1] and eventually comporportionation [2] by taxonomically diverse microorganisms. Tightly interwoven with carbon, nitrogen and metal cycles, the sulfur cycle is tied to both cellular and ecosystem level-processes [3]. In marine sediments, sulfate is an ubiquitous electron acceptor and sulfate-reducing microorganisms have been estimated to contribute to up to $29 \%$ of the organic matter remineralisation [4]. Sulfate reducers can generate massive concentrations of sulfides, which in turn serve as an electron donor for symbiotic or free-living sulfur-oxidizing microorganisms that recycle sulfides into sulfate [5].

Although sulfate reduction and sulfide oxidation have received most attention, our knowledge of the identity of microorganisms and metabolic pathways involved in these processes is limited and novel lineages of microorganisms mediating steps in the sulfur cycle remain to be discovered $[6,7]$.

Furthermore, sulfur cycling is highly complex; sulfur transformations by microorganisms and the geochemical reactivity of reduced sulfur molecules with metal oxides generate several inorganic sulfur compounds of intermediate oxidation states (e.g., thiosulfate, $\mathrm{S}_{2} \mathrm{O}_{3}{ }^{2-}$; tetrathionate, $\mathrm{S}_{4} \mathrm{O}_{6}{ }^{2-}$; sulfite, $\mathrm{HSO}_{3}{ }^{-}$; polysulfides: $\mathrm{S}^{2-}{ }_{\mathrm{n}+1}$ ). These inorganic $\mathrm{S}$ compounds are all substrates for further microbial oxidation, reduction or disproportionation $[3,8]$ and precipitation with reduced metals [9]. The rapid recycling of these sulfur species has biogeochemical significance, especially in low sulfate environments, where the rapid turn-over of these compounds provides an opportunity for shortcuts in the sulfur cycling and potentially sustains a large variety of microorganisms [10]. However, because efficient microbial scavenging drives their concentrations down to the picomolar range, the importance of these processes remains unrecognized. An alternative strategy to reveal these processes is to look for genomic evidences of the identity, ecology and functional properties of microbes metabolizing these compounds.

The genetic underpinning of sulfur transformations is still poorly resolved, and is complicated by the bidirectional activities of key enzymes, and by the diversity and complexity of many enzymatic pathways. For example, the metabolic pathways involved in sulfur compounds disproportionation remains unknown and even the exact mechanisms involved in sulfide oxidation remain little understood [1, 11]. Another important, yet largely overlooked component of the sulfur cycle involves the utilisation and formation of organo-sulfur molecules (OSM). These labile metabolites including sulfonate (compounds with a $\mathrm{R}^{-\mathrm{SO}_{3}}{ }^{-}$ functional group) and sulfonium such as dimethylsulphoniopropionate (DMSP) are produced by macroand micro-algae and may represent an important source of sulfur for certain microorganisms in freshwaters $[12,13]$ and oceans, where genetic potential for transformation of OSM is widespread in marine bacteria [14].

Due to the abundance of sulfur compounds in sea water, much of our knowledge on sulfur cycle comes from marine sediments, which constitute a major biotope for sulfur cycling microorganisms [3]. However, the distribution of sulfur cycling microorganisms is not limited to marine environments, and sulfur cycling 
microorganisms also have important ecological roles in sulfate-poor environments such as wetlands and lakes $[7,15]$.

Marine-derived lakes are natural laboratories for understanding the sulfur cycle. In the polar regions, melting ice sheets lead to an isostatic rebound of the continents, isolating fjords that become marinederived lakes. Melting snow and glaciers then discharge freshwater into these lakes that floats on the denser seawater. This density gradient is re-enforced by a mostly permanent ice cover, resulting in meromictic lakes that are layered physically and chemically with well-defined environments, enabling the development of complex and stable microbial communities along light, nutrient and redox gradients [16]. The water column of these lakes is divided into a mixolimnion consisting of the oxic surface freshwater layer immediately beneath the ice, and an anoxic and sulfidic monimolimnion, derived from the marine bottom layer. The chemocline at the interface between these layers is a zone of highest chemical reactivity in the lake, typically associated with elevated microbial activity and intense sulfur cycling [17, 18]. Phototrophic or chemotrophic sulfur oxidizers that recycle sulfides produced in the anoxic monimolimnion overlap or coexist in the chemocline, depending on oxygen and light penetration [19]. The activity of these microorganisms results in the production of sulfate and sulfur deposits outside the cells, leading to yellow or orange coloration of the water [20]. In addition, metal oxides (iron and manganese) frequently accumulate in meromictic lakes [21], leading to chemical oxidation of sulfur species.

The broad range of physico-chemical conditions in a single water column makes polar meromictic ecosystems ideal lake-size laboratories to investigate sulfur-cycling microorganisms and metabolic pathways. We hypothesized that sulfur cycle intermediate (SCl) molecules such as thiosulfate, tetrathionate, sulfite and polysulfides are a major source of energy for aquatic microbial communities, and that related biogeochemical processes would be evident in a polar meromictic lake because of the prolonged selection of microbial species and functions across stable biogeochemical gradients.

To test this hypothesis, we analysed the microbial community composition, abundance and metabolic potential in Lake $\mathrm{A}\left(83^{\circ} 00^{\prime} \mathrm{N}, 75^{\circ} 30^{\prime} \mathrm{W}\right)$, a deep marine-derived meromictic lake in the Canadian High Arctic (Fig. 1a), with a focus on sulfur cycling microorganisms across light and redox gradients. Applying genome-centric metagenomics, we recovered a large variety of sulfur cycling microorganisms with contrasted and complex metabolic pathways involved in sulfur transformations. We elucidated the importance of sulfur cycle intermediates as well as organic sulfur molecules in structuring the microbial community composition and function over the broad range of geochemical conditions that are represented in this ecosystem.

\section{Results}

Oxygen, salinity and temperature profiles of Lake A, measured at the sampling time (18 July 2017), showed that the water column under the ice was highly stratified, with similar profiles to previous years [21]. The freshwater oxygenated mixolimnion extended to the chemocline, located from 12 to $24 \mathrm{~m}$ below the upper ice surface ice, and marine-derived saline, anoxic water occurred below 24 meters (Fig. 1b). 


\section{Microbial Community Composition}

Microbial community composition and abundance was investigated by 16S rRNA (rRNA) and 16S rRNA gene ( $\mathrm{rDNA}$ ) sequencing and quantification (qPCR) down the water column with discreet samples from $2 \mathrm{~m}, 6 \mathrm{~m}, 14 \mathrm{~m}, 22 \mathrm{~m}, 34 \mathrm{~m}, 40 \mathrm{~m}, 55 \mathrm{~m}, 65 \mathrm{~m}$. In addition, 16S rDNA sequences were extracted from the metagenome dataset and analyzed along with the amplicon data (Fig. 1d). Bacteria represented nearly $100 \%$ of the 16 S rRNA genes in both qPCR and metagenomic data in the upper freshwater samples and $90 \%$ in the saline water samples (Supplementary Figure S1), therefore only the bacterial community is detailed in this study. The bacterial community compositions of replicate samples were very similar (Fig. 1c and Supplementary Figure S1). Although the number of rRNA genes may fluctuate depending on species and physiological state, taxonomic profiles from 16S rRNA and 16S rDNA were congruent, suggesting that these communities were likely active (Supplementary Figure S2). The results were also congruent with the microbial community composition recovered from metagenomic data with the exception of SAR11 and Patescibacteria lineages, which appeared to be underestimated by amplicon sequencing (Fig. 1c and Supplementary Figure S2).

The different depth strata of the lake had sharply contrasting community structures (Non-Parametric Multivariate ANalysis of VAriancs, NPMANOVA, p:0.0001, Figs. 1c and 1d). The bacterial community from the $2 \mathrm{~m}$ sample collected immediately beneath the ice, was dominated by Flavobacteriales (39\% of the 16S rRNA metagenomic reads), Burkholderiales (10\%) and Actinobacteria $\mathrm{Hgcl}(13 \%)$. At $6 \mathrm{~m}$, Cyanobacteria (12\%), Verrucomicrobia (Chtoniobacteraceae, 14\%), and Actinobacteria Hgcl (16\%) were predominant. At the freshwater-saline transition (14 m), the proportion of Alphaproteobacteria (7\%), including SAR11, Planctomycetes (4\%) and Bacteroidetes (10\%) increased. At the bottom of the oxicanoxic transition zone (22 m), the microbial community was dominated by Alphaproteobacteria (13\%), including members of the Rhodospirillalles (17\%), Marinimicrobia, also known as SAR406 (14\%), Chlorobiaceae (6\%) and Cyanobacteria (4\%). In the anoxic saline strata of the lower water column $(34,40$, 55 and $65 \mathrm{~m}$ ), the microbial community was mainly dominated by Chloroflexi (up to $19 \%$ at $55 \mathrm{~m}$ ), Desulfobacteraceae, including the SEEP SRB1 lineage (10\%), Atribacteria (up to $9 \%$ at $40 \mathrm{~m}$ ), Patescibacteria (up to $15 \%$ at $34 \mathrm{~m}$ ) and Omnitrophicaeota (up to $12 \%$ at $65 \mathrm{~m}$ ). The proportion of unclassified reads also increased with depth, from only $4 \%$ of the reads in surface water to $40 \%$ at $65 \mathrm{~m}$ in the $16 \mathrm{~S}$ amplicon datasets (Supplementary Figure S1). However, up to $28 \%$ of the unclassified reads of the $65 \mathrm{~m}$ samples were related to a single Operational Taxonomic Unit (OTU, 97\% similarity) distantly related ( $96 \%$ sequence similarity) to a sequence recovered from the Okinawa Trough. Quantification of $16 \mathrm{~S}$ rRNA genes indicated a relatively constant bacterial abundance throughout the water column, with on average $1.36 \pm 0.6 \times 10^{4} 16 \mathrm{~S}$ rRNA genes $\mathrm{ml}^{-1}$, except for the oxic-anoxic transition zone where bacterial abundance reached $5.33 \times 10^{4} 16 \mathrm{~S}$ rRNA genes $\mathrm{ml}^{-1}$ (Fig. 1d).

\section{Depth Distribution Of Sulfur Cycling Genes}


Shotgun metagenomes from the eight depths were sequenced to evaluate the metabolic potential of Lake A microbial communities. A total of 10378 different genes (KEGG Orthologues) were identified in the metagenomes. Hierarchical clustering of the overall metabolic potentials of the samples was congruent with the clustering based on taxonomic profiles and geochemical data, with two major clusters: the freshwater samples (2, 6 and $14 \mathrm{~m})$ and the saline anoxic samples (34, 40, 55 and $65 \mathrm{~m})$; and the oxycline sample (22 $\mathrm{m}$ ) branching separately on the dendrogram (Fig. 2). A large number of genes involved in sulfur cycling were identified in all samples, and were distributed vertically according to the physicochemical gradients (Fig. 2).

Genes for thiosulfate oxidation (doxD, tsdA, TST, pdo_Rho, soxABCDXYZ) were detected in all freshwater samples (from 2 to $22 \mathrm{~m}$ ). Sulfate (soXABCDXYZ) and sulfite (soe $A B$, suoX) oxidation genes were mainly identified in the most oxygenated freshwater layers, whereas genes for sulfide $(f c c A B)$, and polysulfide ( $S Q R, H d r$-like) oxidation pathways were mainly found in the microoxic conditions of the oxycline waters (14 and $22 \mathrm{~m}$ ) (Fig. 2). Few of these genes were also detected in anoxic waters. By contrast, genes involved in dissimilatory sulfate and sulfite reduction pathways (dsrAB, aprAB, sat, qmo, asrAB), thiosulfate $(p h s C)$ and tetrathionate $(t t r B C$, otr) reductions and polysulfide and elemental sulfur reductions ( $p s r A$, hydABCD, npsr) were mainly detected in saline and anoxic waters. All oxidative and reductive pathways were detected at the bottom of the oxycline (22 m) (Fig. 2).

Genes for the transformation of organic sulfur molecules (sulfonium and sulfonate) were also identified (Fig. 2). Genes involved in degradation of dimethylsulfate ( $d d d L Q K P W, d m d A)$, methanesulfonate $(m s m A)$ and other sulfonates, such as cysteine (cuyAZ), sulfopropanediol $(h s p N)$, taurine $(\operatorname{tau} A)$, or sulfoacetaldehyde $(X S C)$ were detected throughout the water column but in higher proportion in freshwater. By contrast, genes coding dimethyl sulfoxide reductases ( $d m s A B C$ ) were mainly found in anoxic saline waters (Fig. 2). Genes for sulfatases (betC and ars/A), catalysing hydrolysis of sulfate esters, were abundant throughout the water column, with higher numbers at the bottom of the oxycline (22 m) (Fig. 2).

\section{Metagenome Assembled Genomes Of S-cycling Populations}

A total of 250 genomic bins with $>50 \%$ completeness and $<5 \%$ contamination levels were recovered from the combined metagenomic dataset. Among them, 154 genomic bins (61.4\%) harbored genes for sulfur cycling (Fig. 3). Taxonomic affiliation of these bins, inferred from 16S rRNA gene and other ribosomal protein sequences, indicated a large diversity of sulfur cycling microorganisms representing most of the predominant lineages identified in the water column by $16 \mathrm{~S}$ rDNA approaches. Bacteroidetes (21 different bins), Alphaproteobacteria (17 bins), Parcubacteria (17 bins), Omnitrophicaeota (12 bins), Chloroflexi (12 bins), Actinobacteria (11 bins), Deltaproteobacteria (9 bins), Planctomycetes (9 bins) and Marinimicrobia (8 bins) were the most represented lineages in bins with sulfur transformation genes (Fig. 3). 


\section{Sulfur-oxidizing populations}

The potential for thiosulfate oxidation was widespread in the community (Figs. 3 and 4). Numerous Parcubacteria, Actinobacteria and Chloroflexi bins included genes for the thiosulfate:quinone dehydrogenase ( $\operatorname{dox} D)$, while the gene for the thiosulfate dehydrogenase ( $t s d A$ ) was mainly detected in Alphaproteobacteria and Bacteroidetes. Genes of persulfide dioxygenase fused with a rhodanese domain (pdo_rho), potentially involved in thiosulfate oxidation [22] were widespread in the Bacteroidetes and Planctomycetes bins (Fig. 3). The SoxABCDXYZ complex for thiosulfate oxidation was also identified in Alpha- and Beta-proteobacteria (Fig. 3). This complex was coupled with oxidative DsrAB genes in Alphaproteobacteria, indicating potential for $\mathrm{H}_{2} \mathrm{~S}$ oxidation to sulfate. Oxidative DsrAB genes and genes for sulfide $(f c c A B)$ and sulfur oxidation through the sulfide:quinone oxidoreductase (SQR) were identified in the Chlorobiaceae as well as in few Alphaproteobacteria bins. Betaproteobacteria bins harbored genes for sulfide oxidation (soeAB and sorB). SQR gene was also detected in Cyanobacteria, Actinobacteria, Bacteroidetes, Gamma- and Deltaproteobacteria. Sulfur and thiosulfate oxidation through Hdr-like complex [23] was identified in one alphaproteobacterial bin.

\section{Sulfur-reducing populations}

Potential for thiosulfate reduction through thiosulfate reductase (PhsC gene) was identified in Bacteroidetes bins recovered from anoxic samples (Figs. 3 and 4). Tetrathionate reductase genes (ttrBC) were mainly found in Rhodospirillales bins while genes coding the octoheme tetrathionate reductase (otr) were detected in few Desulfobacteraceae, Anaerolineae, Chlorobiaceae and Bacteroidetes bins. The sulfate reduction pathway (DsrAB, AprAB, Sat and Qmo genes) was identified in Deltaproteobacteria bins as well as in one Chloroflexi, two Planctomycetes and three Parcubacteria (Candidatus Nealsonbacteria, $\mathrm{Ca}$. Zixibacteria and $\mathrm{Ca}$. Abyssubacteria) bins recovered from the saline anoxic waters (Figs. 3 and 4). Anaerobic sulfite reductase genes (asr $A B$ ) were detected in all Omnitrophica bins as well as in two Planctomycetes and two Parcubacteria bins. Polysulfide reductase genes ( $p s r A$ ) were found in Deltaproteobacteria as well as in some Marinimicrobia and Bacteroidetes bins. Sulfhydrogenase genes (hydABCD), involved in $\mathrm{S}^{0}$ and polysulfide reduction were also identified in genomic bins. However, while HydACD genes were detected in 8 Parcubacteria bins, including $\mathrm{Ca}$. Kuenenbacteria, the gene coding the beta subunit ( $h y d B$ ) was detected only in half of these bins. By contrast hydB alone was also detected in Omnitrophica, Marinimicrobia and WOR1 (Ca. Saganbacteria) bins (Fig. 3). NADH-dependent persulfide reductase genes (npsr), involved in persulfide, polysulfide or $\mathrm{S}^{0}$ reduction were identified in Deltaproteobacteria, Parcubacteria (Ca. Abyssubacteria and Candidate Division KSB1) as well as in two Planctomycetes bins. A fusion of DsrE, Npsr, and TusA genes and including two rhodanese regions was also identified in the metagenomic sequences and in two bins related to $\mathrm{Ca}$. Nealsonbacteria and Phycisphaeraceae (Fig. 3), suggesting a novel gene. Rhodanese regions and TusA protein are involved in sulfur binding and intracellular transport while both DsrE and Npsr are involved in sulfur reduction, suggesting a role in intracellular elemental sulfur reduction for this new gene.

\section{Organic sulfur utilizing populations}


A metabolic potential for organic sulfur utilisation was detected in 87 bins, mainly related to proteobacterial lineages, that were recovered throughout the water column (Fig. 4). The potential for transformation of dimethylsulfoniopropionate (DMSP) into dimethylsulfide (DMS) was detected in Alphaproteobacteria bins, including SAR11/Pelagibacter bin (Figs. 3 and 4). By contrast, genes for anaerobic dimethylsulfoxide reductase $(\mathrm{dms} A B)$ were identified in Deltaproteobacteria, Chloroflexi and few Bacteroidetes bins. The metabolic potential for sulfonate degradation, including genes coding for enzymes involved cysteine (cuyAZ), sulfopropanediol ( $h s p M)$, taurine (tauA), and sulfoacetaldehyde (xsc) catabolism, was mainly found in Actinobacteria, Planctomycetes and Alpha- and Beta-proteobacteria bins (Figs. 3, 4 and 5). Multiple (more than 10) sulfatase genes were identified in some Planctomycetes, Bacteroidetes, Alphaproteobacteria and Chloroflexi bins.

\section{Microbial Network For Sulfur Metabolism}

Based on the identified genes, metabolic capabilities of the Lake A populations were inferred to determine which S-containing molecules were potentially produced or consumed throughout the water column (Fig. 5). Up to 100 different bins (65\% of the bins with S -cycling genes) were predicted to be associated with hydrogen sulfide production or consumption. Metabolic potentials involving the production or consumption of polysulfides ( 89 bins, $58 \%$ ), thiosulfate ( 72 bins, $47 \%$ ) and tetrathionate (61 bins, $40 \%$ ) were also widespread in the Lake A community (Fig. 5). Metabolic potential associated with sulfite and sulfate was less represented in the community with only 46 bins (30\%) associated with sulfite and 30 bins (19\%) with sulfate (Fig. 5).

\section{Discussion}

Ice-capped, the meromictic Lake $A$ is an extreme microbial ecosystem, where strong and persistent environmental gradients provide a natural model for broader understanding of aquatic biogeochemical cycles. The water physico-chemical stratification profile measured in 2017 in the Lake A has been observed since 1974 [18-20], indicating a highly stable system and allowing extrapolation of geochemical profiles from historical data. Based on a previous complete geochemical characterization of the lake waters [21] high concentrations of sulfate occur in the chemocline and in the anoxic waters with increasing sulfide concentrations with depth (Fig. 1). High manganese concentrations were also detected at the chemocline while a peak of iron was observed few meters below (Fig. 1 [21]). Finally, at similar snow and ice cover, photosynthetically available radiation (PAR) was detected down to 20 meters (Fig. 1 [24]). These extrapolations were supported by the depth distribution of green sulfur bacteria (Chlorobium), that confirmed light and sulfides transition zones around 24 meters. Together these observations indicate a stable and intense redox gradient throughout the water column for microbial selection and growth.

RNA and DNA-based 16S amplicon and metagenomic sequencing from the High Arctic Lake A waters revealed multiple contiguous layers of complex yet stable and potentially active microbial communities, 
with putative metabolism aligning with the geochemical gradients of the lake (Fig. 1). The microbial community of the oxic mixolimnion beneath the ice was consistent with cold freshwater communities, with lineages of the Verrucomicrobia, Bacteroidetes, Actinobacteria, Cyanobacteria and Betaproteobacteria, which are frequently observed in lakes and rivers [25-27]. At the chemocline, alphaproteobacterial chemotrophic sulfur oxidizers and phototrophic sulfur oxidizers (Chlorobiaceae), both previously observed in microbial surveys of Antarctica $[17,28]$ and temperate meromictic lakes [19, 29] co-occurred since the lower depth limits of the Lake A photic and aerobic zones coincided (Fig. 1). The chemocline microbial community also shared major similarities with marine communities with, for example, strong proportions of Marinimicrobia (SAR406), Pelagibacter(SAR11) and Deltaproteobacteria SAR324, which are frequently detected in seawater, hadal waters in the deep ocean and oxygen minimum zones [30-32]. By contrast, the microbial community of the saline anoxic monimolimnion showed homologies with deep-sea hypersaline anoxic basin communities, notably with sequences related to Chloroflexi MSBL5, Desulfobacteraceae MSBL7, Planctomycetes MSBL9 and Cloacimonadales MSBL8 [33]. At these depths, the microbial community also shared similarities with anoxic and sulfidic marine sediments, where Deltaproteobacteria SEEP SRB1 and Desulfarculaceae, Atribacteria, Omnitrophica and Chloroflexi members also flourish [34]. Taken together these results reveal that, cascading along its geochemical gradients, the Lake A water column hosts a panoply of microorganisms, relevant to a broad range of environments and environmental conditions from oxic freshwaters to anoxic marine sediments.

\section{New Microbial Agents And Metabolic Pathways For Sulfur Transformation}

From surface layers to the bottom, most of the genomic bins $(61.4 \%)$ recovered from Lake A included genes for sulfur cycling (Figs. 3 and 4). Furthermore, bacterial abundance in Lake A was correlated with the average number of metabolic pathways for sulfur transformation per bin $\left(R^{2}=0.69, p=0.04\right)$, supporting the notion that sulfur cycle represents a major process in Lake A waters and involves a large diversity of microorganisms. Reconstruction of genomic bins highlighted that in addition to the conventional taxa associated with the classical sulfur cycle in meromictic saline lakes such as sulfatereducing Desulfobacteraceae, sulfur-oxidizing Alphaproteobacteria and phototrophic sulfur oxidizing Chlorobiaceae [17], various lineages with poorly known ecological functions are also involved in sulfur transformations. Among these lineages, key genes of sulfur metabolism were identified in Ca. WOR1, SAR86, Lentisphaerae, Aminicentantes, Marinimicrobia, Calditrichaeota, Omnitrophica and Parcubacteria, thereby expanding the known diversity of sulfur cycling bacteria (Fig. 3 and Supplementary Table S1).

A strong functional redundancy in sulfur transformation pathways was detected throughout the water column, with taxonomically diverse microorganisms having similar metabolic pathways (Figs. 3 and 4). For example, sulfide oxidation potential through SQR and the SoxABCXYZ complex was identified in Alphaproteobacteria (Rhodospirillales) and Gammaproteobacteria SAR86. Oxidative DsrAB genes were also identified in half of the Rhodospirillales bins and in the Deltaproteobacteria SAR324, while the Hdrlike complex, also involved in sulfide oxidation was discovered in another Alphaproteobacteria bin, 
congruent with experimental evidence in the Alphaproteobacterium Hyphomicrobium denitrificans [23]. In addition, the SoXABCXYZ complex coupled with SoeAB genes were detected in Betaproteobacteria bins whereas SQR, FCCAB and the oxidative DsrAB genes were ascertained in the Chlorobiaceae bin [28] (Fig. 3). These multiple pathways for sulfide and sulfite oxidations accumulated in the freshwater and chemocline layers (Fig. 4), suggesting that sulfides sustain multiple ecological niches in aquatic environments over space and/or time. If the occurrence of these various sulfur-oxidizing pathways and lineages at the chemocline is supported by the chemical profiles, their identification in the upper freshwater samples, coupled with the co-detection of sulfonate degradation genes (Fig. 2) suggests that organic sulfur molecules may also support sulfur-oxidizing populations in non-sulfidic waters, multiplying the availability of ecological niches and allowing functional redundancy.

In the anoxic saline layer, the dissimilatory sulfate reduction pathway (Sat, Qmo, AprAB and DsrAB genes) occurred in the Deltaproteobacteria bins as expected [7], but was also found in genomic bins affiliated with Chlorolexi, Planctomycetes, Calditrichaeota and Parcubacteria (Ca. Nealsonbacteria, Ca.

Abyssubacteria and $\mathrm{Ca}$. Zixibacteria) (Fig. 3). These results provide an ecological context for these new lineages of sulfate reducers, that were previously proposed after mining of combined metagenomic datasets [6]. Our metagenomic survey also predicted a sulfite reduction potential (AsrAB genes) for Omnitrophica members (Figs. 3 and 4), supporting data mining [6], as well as for few Planctomycetes and Patescibacteria populations in the sulfidic waters of the monimolimnion.

Patescibacteria, Planctomycetes and Chloroflexi phyla showed the strongest variability of genomic potential within their lineages (Figs. 3 and 4). Each of these phyla included populations predicted to gain energy from thiosulfate oxidation, sulfate and sulfite reduction as well as polysulfide/elemental sulfur reduction or oxidation. A new fusion gene probably involved in elemental sulfur/polysulfide reduction was also identified in two genomic bins affiliated with Patescibacteria and Planctomycetes phyla. Sequence comparison with public databases indicated that this gene is also present in a single-cell genome related to the Planctomycetes-derived phylum of the Kiritimatiellaeota, isolated from a deep continental microoxic subsurface aquifer [35], suggesting that this gene might be relevant in microoxic conditions. Interestingly, Planctomycetes, Chloroflexi and Ca. Nealsonbacteria (Patescibacteria) genomic bins also included numerous genes (>10 per bin) coding for sulfatases. These hydrolytic enzymes potentially release sulfate from sulfated organic matter [36], providing additional electron acceptors throughout the water column. Together these results extend the diversity of sulfur cycling microorganisms and metabolic pathways. They suggest new fundamental roles in sulfur cycling for members of the Patescibacteria, Planctomycetes and Chloroflexi in aquatic environments, with strong ecological niche differentiation within member of these lineages.

\section{Utilization Of Sulfur Cycle Intermediates}

Sulfur cycle intermediates (SCls: thiosulfate, tetrathionate, sulfite, polysulfides, elemental sulfur) have a large biogeochemical significance in anoxic and marine environments, creating shortcuts around the 
classic sulfur cycle $[1,8]$. The potential for oxidation and reduction of these sulfur molecules was widespread in the Lake A microbial community, with taxonomically diverse lineages potentially using SCls as electron donors or acceptors (Fig. 3). The number of genes for SCl metabolism and sulfate reduction was similar, suggesting that $\mathrm{SCl}$ utilisation might represent a quantitatively important process in Lake A sulfur cycling (Fig. 2). Furthermore, the number of genomic bins with $\mathrm{SCl}$ utilization genes exceeded the number of bins with sulfate reduction and hydrogen sulfide oxidization pathways and SCls were found as major hubs in the sulfur metabolic network (Fig. 5), indicating a wide diversity of microorganisms able to process SCls.

The potential to use SCls was shared between specialists that use only a limited range of these molecules, and generalists that could potentially metabolise a broad range of sulfur compounds including sulfate or hydrogen sulfide. The specialists included some members of the Parcubacteria with the potential limited to thiosulfate oxidation, Omnitrophica with only genes for sulfite reduction and Bacteroidetes populations with the metabolic potentials for thiosulfate and polysulfide oxidation. By contrast, generalists were mainly represented by members of the Deltaproteobacteria or Alphaproteobacteria lineages with a large suite of sulfur transformation genes, suggesting high variability in substrate utilization (Fig. 4).

The taxonomically diverse microorganisms observed here are likely fuelled by microbial phototrophic and chemotrophic hydrogen sulfide oxidations that generate SCls of various oxidation states [8], as well as by abiotic oxidation of hydrogen sulfide with iron and manganese oxides present in elevated concentrations in the Lake A (Fig. 1)[1]. Although SCls have not been measured in Lake A, a sulfidic smell and a yelloworange color of the water below $22 \mathrm{~m}$ was detected during sampling supporting the presence of polysulfides and aqueous elemental sulfur in the water and the metabolic potentials detected in metagenomic dataset. Together these results indicated a strong ecological role for SCls by providing an energy source for a diverse and abundant microbial community in both fresh, brackish and saline waters.

\section{Organic Sulfur Molecules As Sci Sources}

Oxidized organic sulfur molecules, such as sulfonate and sulfonium are produced by the phytoplankton as osmoprotectants, antioxidants [37] or predator deterrents in aquatic environments [38], and are an important source of sulfur and carbon for pelagic bacteria in the ocean [14]. These compounds are also frequently detected in metabolomes of diatoms [39]. Eukaryotic microalgae were detected in the oxic mixolimnion in the metagenomic dataset (e.g., Chrysophyceae, Ochrophyta, Chlorophyceae, data not shown) and in a previous amplicon survey [24], therefore the presence of eukaryotic sulfur metabolites in the Lake A would not be surprising. The genetic capacity for OSM degradation was widely distributed, occurring in $56 \%$ of the genomic bins (Figs. 3, 4 and 5). Proteobacterial lineages were detected as major sulfonate degraders in the mixolimnion and chemocline. Since these degradation processes release sulfite in the water, our results suggest that organic sulfur molecules might have an important ecological 
role, providing sulfur compounds of intermediate oxidation states in aerobic and microaerobic aquatic systems regardless of the salinity and sulfate concentration.

Genes for DMSP utilisation, identified in the freshwater and brackish waters of the lake were also detected in Alphaproteobacteria (Rhodospirillales and SAR11/Pelagibacter bins) and Actinobacteria (Acidimicrobiia), as reported in surface oceans [14]. In addition, numerous genes for respiration of dimethylsulfoxide (DMSO) were identified in the anoxic monimolimnion and in Desullfobacteraceae, Chloroflexi and Bacteroidetes bins, suggesting that algal metabolites could sink within senescent phytoplankton from the upper water column and be used as an alternative energy source by anaerobic microbial populations in the lower water column of Lake A.

\section{Conclusions}

Isolated and highly stratified, the High-Arctic meromictic saline Lake A offered an outstanding opportunity to investigate microbial metabolism along oxygen, sulfate, sulfide and salinity gradients. Although the chemocline harbored the taxonomically and functionally most diverse and abundant microbial community, defining this layer as an hotspot for microbial activity and sulfur transformations as in other meromictic lakes $[17,19]$, genes for sulfur transformations were identified throughout the water column, and provided new insights into sulfur cycling microorganisms over a large range of environmental conditions. The pathways and taxa involved in sulfide oxidation and sulfate reduction were complex and diverse. However, the metagenomic dataset revealed that sulfur transformations were not limited to these classic processes and that multiple sulfur cycling pathways could be simultaneously operating throughout the water column, with taxonomically diverse populations using sulfur cycle intermediates as electron donors or acceptors. Genes for organic sulfur molecule degradation and respiration were also abundant and widely distributed in the microbial community, suggesting that phytoplankton metabolites might also be a major source of energy for freshwater and marine bacteria. Our data extend the diversity of sulfur cycle lineages and metabolic pathways in aquatic ecosystems and emphasize the ecological importance of sulfur cycle intermediates as key hubs for electron flow and energy production over a wide range of environmental conditions.

\section{Methods}

\section{Sample Collection And Nucleic Acid Extraction}

In summer 2017 (18 July 2017), three independent $24 \mathrm{~cm}$-diameter holes were drilled through the ice ( $0.6 \mathrm{~m}$ thickness) near the middle of the Lake A (Ellesmere Island, $82^{\circ} 59.667^{\prime} \mathrm{N}, 75^{\circ} 26.602^{\prime} \mathrm{W}$; Fig. $1 \mathrm{a}$ ). Oxygen concentration, salinity and temperature profiles throughout the water column were measured using a XR-420 CTD profiler (RBR Ltd., Ottawa, Canada) to $65 \mathrm{~m}$, which was the length of the instrument cable, to determine sampling depth. Eight sampling depths $(2 \mathrm{~m}, 6 \mathrm{~m}, 14 \mathrm{~m}, 22 \mathrm{~m}, 34 \mathrm{~m}, 40 \mathrm{~m}, 55 \mathrm{~m}$ and $65 \mathrm{~m}$ ) were selected to cover all identified water layers (Fig. 1). A Limnos Water sampler (KC, Denmark), which was previously rinsed with $10 \% \mathrm{HCl}$, then sterile MilliQ water and maintained closed until sampling, 
was lowered into the ice holes down to the sampling depth. Water was visibly orange in the $22 \mathrm{~m}, 34 \mathrm{~m}$, $40 \mathrm{~m}, 55 \mathrm{~m}$ and $65 \mathrm{~m}$ samples. For each selected depth, $1 \mathrm{~L}$ of each triplicate water sample was directly filtered through separate $0.22 \mu \mathrm{m}$ pore size Sterivex filters and then stored below $-50^{\circ} \mathrm{C}$ until nucleic acid extraction.

Nucleic acids (DNA and RNA) of two of the replicates samples per depth were extracted from the same Sterivex filters using Qiagen Allprep DNA/RNA Mini Kit. The Sterivex cartridges were opened and the membrane filters were cut into small pieces before the lysis steps, as previously described [40]. All steps of the nucleic acid extractions, from the opening of the filters to the nucleic acid resuspension in autoclaved, filtered and UV-treated water, were carried out in a sterile laminar flow cabinet. Negative control (no template) of nucleic acids extraction was simultaneously carried out. The DNA extracts were stored at $-20^{\circ} \mathrm{C}$ until library preparation. For RNA extracts, two additional DNase steps (DNase I, Ambion, Foster City, CA, USA) were carried out to remove any trace of carried over DNA. The absence of DNA contamination was confirmed by amplification of $16 \mathrm{~S}$ rRNA genes with bacterial primers using the RNA extracts (undiluted and diluted ten times) as template, with no product detected after 35 PCR cycles. The RNA was then immediately converted to cDNA using a High-Capacity cDNA Reverse Transcription kit (Applied Biosystems, Foster City, CA, USA) and stored as cDNA at $-20^{\circ} \mathrm{C}$ until library preparation.

\section{Quantitative Pcr}

The abundance of bacterial and archaeal 16S rRNA genes was estimated on the two replicate samples per depth using quantitative PCR (qPCR) with primers Bact1369f/Bact1492r [41] and Arc787f/Arc1059r [42], respectively. Quantification was performed in triplicate with a range of template concentrations (0.1, $0.5,1 \mathrm{ng}$ of DNA) to compensate for any PCR inhibition. Genomic DNA extracted from Methylomonas methanica (DSM25384) and Methanosarcina acetivorans (DSM2834) were serially diluted to construct standard curves (concentration ranged from $10^{2}$ to $10^{6} 16 \mathrm{~S}$ rRNA genes per reaction). The $\mathrm{R}^{2}$ of standard curves obtained by qPCR were above 0.99 , PCR efficiencies were above $88.7 \%$, and melting curves showed no trace of non-specific amplifications. Threshold cycle (Ct) of the samples ranged from 15 to 25 cycles whereas Ct of negative controls (water) were all after 37 cycles. The qPCR results were expressed in terms of $16 \mathrm{~S}$ rRNA gene numbers per millilitre of water sample (Fig. 1d).

\section{Illumina Miseq Amplicon Library Preparation, Sequencing And Analysis}

Microbial community composition of the samples was determined by high throughput sequencing of bacterial and archaeal 16S rRNA (CDNA) and 16S rRNA genes (DNA) using primers targeting the bacterial V4-V5 region (S-D-Bact-0516-a-S-18/S-D-Bact-0907-a-A-20; 460 bp product) [43] and the archaeal V1-V3 region (A27F/Arc518R; 500 bp), respectively [44]. All PCR reactions were carried out following [45]. Samples and negative controls of nucleic acids extraction, transcription and PCRs were sequenced using 
an Illumina MiSeq v3 kit at the IBIS/Laval University, Plate-forme d'Analyses Génomiques (Québec, QC). Reads were assembled into single paired-end sequences, curated and clustered into OTUs (97\% sequence similarity) as detailed in a GitHub repository (https://github.com/CruaudPe/MiSeq_Multigenique). OTUs detected in negative controls were removed from the analysis as described [27]. Taxonomic affiliations of the reads were determined with Mothur [46] using BLAST against Silva database release 132 as reference [47].

\section{Metagenomic Library Preparation, Sequencing And Analysis}

One metagenome per sample depth (8 metagenomes) was constructed using a Nextera XT Library Kit (Illumina, San Diego, CA, USA). The 8 metagenomes were pooled equimolarly then sequenced in two Illumina MiSeq $(2 \star 300 \mathrm{bp})$ runs and one Illumina NexSeq run $(2 * 150 \mathrm{bp})$ at the Institut de Biologie Integrative et des Systèmes (IBIS) sequencing platform (Univeristé Laval, Canada) and at the CGEB Integrated Microbiome Resource (Dalhousie University, Canada) respectively. Datasets were quality filtered using the Trimmomatic tool [48], with default settings. Paired-end joining was done using FLASH2 [49]. The 16S rRNA reads longer than 110 bp were isolated from metagenomic reads using REAGO 1.1 [50], and taxonomic assignments were performed as for the 16S rRNA gene amplicons.

Each metagenome was assembled separately from paired-end reads passing quality filtering using SPAdes [51]. Assembled contigs and mapping files (BAM files generated using BBmap) were uploaded to the Department of Energy Joint Genome Institute (DOE-JGI) IMG/MER analysis pipeline [52] for gene calling and functional annotation. To account for differences in sequencing depth between samples, metagenomes were normalized to the size of the smallest dataset.

\section{Binning And Functional Characterization}

For metagenome assembled genome reconstruction, all quality filtered sequences were pooled and coassembled using MEGAHIT [53]. Read coverage of the contigs was carried out using bwa-mem (http://bio-bwa.sourceforge.net), followed by contig binning using MetaBAT-2 [54] with contigs longer than $2000 \mathrm{bp}$. The completeness and contamination level of the combined genomic bins were then evaluated using CheckM [55]. Only bins with a contamination level under $5 \%$ and completeness above $50 \%$ were analysed. Genetic composition of genomic bins was then explored using KEGG [56] and MetaCyc [57] pathway mappers with genes identified by IMG/MER in the co-assembly. The results were manually checked and the presence of specific pathways was determined by detection of key genes as detailed in the Supplementary Material.

\section{Statistical analysis}

Statistical analyses of the data set (Student t-test, Non-Parametric Multivariate Analysis Of VAriance (NPMANOVA), Bray-Curtis-based dissimilarity index calculations and correlation-based clustering, Non 
Metric Multidimensional Scale (NMDS)) were carried out according to recommendations of the Guide to Statistical Analysis in Microbial Ecology [58], using PAST software [59].

\section{Abbreviations}

$S$

Sulfur

OSM

Organo-Sulfur Molecules

$\mathrm{SCl}$

Sulfur Cycle Intermediate

DMS

Dimethylsulfide

DMSP

Dimethylsulfoniopropionate

DMSO

Dimethylsulfoxide

OTU

Operational Taxonomic Unit

qPCR

quantitative Polymerase Chain Reaction

NMDS

Non-Metric Multidimensional Scaling

NPMANOVA

Non-Parametric Multivariate Analysis Of VAriance

MSBL

Mediterranean Sea Brine Lake

Ca.

Candidatus

BLAST

Basic Local Alignment Search Tool

PAR

Photosynthetically Available Radiation

\section{Declarations}

\section{Ethics statement}

All necessary permits were obtained for this study, specifically a Nunavut Research License for research in Nunavut, and a Parks Canada Research and Collection Permit, for sampling in Quttinirpaaq National 
Park.

\section{Consent for publication}

Not applicable.

\section{Availability of data and materials}

Assembled metagenome data are available in IMG/MR (https://img.jgi.doe.gov/mer/) under the following accession numbers: 3300033443, 3300033444, 3300033445, 3300033439, 3300033411, 3300033473, 3300033474,3300033495 . Co-assembly is also available on IMG/MR under accession number 3300033064. Raw amplicon sequences and bin files were deposited in the NCBI public database under Bioproject PRJNA616293 (https://www.ncbi.nlm.nih.gov/bioproject/PRJNA616293). In house scripts used in this study are available on GitHub/CruaudPe. Environmental metadata were previously published [21] and additional data are available in the Nordicana $D$ database (http://www.cen.ulaval.ca/nordicanad).

\section{Competing interests}

Authors declare no competing interest

\section{Funding}

This work was supported by the program Sentinel North financed by the Canada First Research Excellence Fund (CFREF); the Natural Sciences and Engineering Research Council of Canada (NSERC); the CNR (Italy) - Universite Laval UMI-MicroMeNu program; the fond de Recherche du Quebec Nature et Technologies (FQRNT); and the Network of Centres of Excellence ArcticNet.

\section{Authors' contributions}

A.V. and W.F.V. designed the research, W.F.V. and A.I.C obtained funding, logistics and permits for the research; A.I.C. and W.F.V. conducted the field sampling; A.V. generated the molecular data; A.V. and P.C. analyzed the data; A.V. wrote the paper with W.F.V., C.L., P.C., R.-M.C. and A.I.C.

\section{Acknowledgements}

We thank Denis Sarrazin (Centre d'études nordiques) and members of the Culley Lab for assisting the sampling effort, Polar Continental Shelf Program and Canadian Helicopter Ltd. for the logistic support, 
and Calcul Quebec for computing resources and technical support.

\section{References}

1. Jørgensen BB, Findlay AJ, Pellerin A. The biogeochemical sulfur cycle of marine sediments. Front Microbiol. 2019;10:849.

2. Amend JP, Aronson HS, Macalady J, LaRowe DE. Another chemolithotrophic metabolism missing in nature: sulfur comproportionation. bioRxiv. 2019;820597.

3. Wasmund K, Mußmann M, Loy A. The life sulfuric: microbial ecology of sulfur cycling in marine sediments. Environ Microbiol Rep. 2017;9:323-44.

4. Bowles MW, Mogollón JM, Kasten S, Zabel M, Hinrichs K-U. Global rates of marine sulfate reduction and implications for sub-sea-floor metabolic activities. Science. 2014;344:889.

5. Sievert SM, Hügler M, Taylor CD, Wirsen CO. Sulfur oxidation at Deep-Sea Hydrothermal Vents. In: Dahl C, Friedrich CG, editors. Microb Sulfur Metab. Berlin Heidelberg: Springer; 2008. pp. 238-58.

6. Anantharaman K, Hausmann B, Jungbluth SP, Kantor RS, Lavy A, Warren LA, et al. Expanded diversity of microbial groups that shape the dissimilatory sulfur cycle. ISME J. 2018;12:1715-28.

7. Vigneron A, Cruaud P, Alsop E, de Rezende JR, Head IM, Tsesmetzis N. Beyond the tip of the iceberg; a new view of the diversity of sulfite- and sulfate-reducing microorganisms. ISME J. 2018;12:2096-9.

8. Findlay AJ, Kamyshny A. Turnover rates of intermediate sulfur species (Sx2-, S0, S2032-, S4062-, SO32-) in anoxic freshwater and sediments. Front Microbiol. 2017;8:2551.

9. Rickard D, Morse JW. Acid volatile sulfide (AVS). Mar Chem. 2005;97:141-97.

10. Jørgensen BB. A Thiosulfate shunt in the sulfur cycle of marine sediments. Science. 1990;249:152.

11. Wang R, Lin J-Q, Liu X-M, Pang X, Zhang C-J, Yang C-L, et al. Sulfur oxidation in the acidophilic autotrophic Acidithiobacillus spp. Front Microbiol. 2019;9:3290.

12. Fakhraee M, Li J, Katsev S. Significant role of organic sulfur in supporting sedimentary sulfate reduction in low-sulfate environments. Geochim Cosmochim Acta. 2017;213:502-16.

13. Couture R-M, Fischer R, Van Cappellen P, Gobeil C. Non-steady state diagenesis of organic and inorganic sulfur in lake sediments. Geochim Cosmochim Acta. 2016;194:15-33.

14. Landa M, Burns AS, Durham BP, Esson K, Nowinski B, Sharma S, et al. Sulfur metabolites that facilitate oceanic phytoplankton-bacteria carbon flux. ISME J. 2019;13:2536-50.

15. Hausmann B, Pelikan C, Herbold CW, Köstlbacher S, Albertsen M, Eichorst SA, et al. Peatland Acidobacteria with a dissimilatory sulfur metabolism. ISME J. 2018;12:1729-42.

16. Cavicchioli R. Microbial ecology of Antarctic aquatic systems. Nat Rev Microbiol. 2015;13:691-706.

17. Lauro FM, DeMaere MZ, Yau S, Brown MV, Ng C, Wilkins D, et al. An integrative study of a meromictic lake ecosystem in Antarctica. ISME J. 2011;5:879-95.

18. Peduzzi S, Tonolla M, Hahn D. Isolation and characterization of aggregate-forming sulfate-reducing and purple sulfur bacteria from the chemocline of meromictic Lake Cadagno, Switzerland. FEMS 
Microbiol Ecol. 2003;45:29-37.

19. Pjevac P, Korlević M, Berg JS, Bura-Nakić E, Ciglenečki I, Amann R, et al. Community shift from phototrophic to chemotrophic sulfide oxidation following anoxic holomixis in a stratified seawater lake. Appl Environ Microbiol. 2015;81:298.

20. Mas J, Van Gemerden H. Storage products in purple and green sulfur bacteria. In: Blankenship RE, Madigan MT, Bauer CE, editors. Anoxygenic Photosynth Bact. Springer Netherlands; 1995. p. 973-90.

21. Gibson JAE, Vincent WF, Van Hove P, Belzile C, Wang X, Muir D. Geochemistry of ice-covered, meromictic Lake A in the Canadian High Arctic. Aquat Geochem. 2002;8:97-119.

22. Motl N, Skiba MA, Kabil O, Smith JL, Banerjee R. Structural and biochemical analyses indicate that a bacterial persulfide dioxygenase-rhodanese fusion protein functions in sulfur assimilation. J Biol Chem American Society for Biochemistry Molecular Biology. 2017;292:14026-38.

23. Koch T, Dahl C. A novel bacterial sulfur oxidation pathway provides a new link between the cycles of organic and inorganic sulfur compounds. ISME J. 2018;12:2479-91.

24. Charvet S, Vincent W, Comeau A, Lovejoy C. Pyrosequencing analysis of the protist communities in a High Arctic meromictic lake: DNA preservation and change. Front Microbiol. 2012;3:422.

25. Tran P, Ramachandran A, Khawasek O, Beisner BE, Rautio M, Huot $Y$, et al. Microbial life under ice: Metagenome diversity and in situ activity of Verrucomicrobia in seasonally ice-covered lakes: Sub-ice Verrucomicrobia genomes in Quebec lakes. Environ Microbiol. 2018;20:2568.

26. Vigneron A, Lovejoy C, Cruaud P, Kalenitchenko D, Culley A, Vincent WF. Contrasting winter versus summer microbial communities and metabolic functions in a permafrost thaw lake. Front Microbiol. 2019;10:1656.

27. Cruaud P, Vigneron A, Fradette M-S, Dorea CC, Culley Al, Rodriguez MJ, et al. Annual bacterial community cycle in a seasonally ice-covered river reflects environmental and climatic conditions. Limnol Oceanogr. 2019;65:21-37.

28. Ng C, DeMaere MZ, Williams TJ, Lauro FM, Raftery M, Gibson JA, et al. Metaproteogenomic analysis of a dominant green sulfur bacterium from Ace Lake, Antarctica. ISME J. 2010;4:1002-19.

29. Yurkova N, Rathgeber C, Swiderski J, Stackebrandt E, Beatty JT, Hall KJ, et al. Diversity, distribution and physiology of the aerobic phototrophic bacteria in the mixolimnion of a meromictic lake. FEMS Microbiol Ecol. 2002;40:191-204.

30. Hawley AK, Nobu MK, Wright JJ, Durno WE, Morgan-Lang C, Sage B, et al. Diverse Marinimicrobia bacteria may mediate coupled biogeochemical cycles along eco-thermodynamic gradients. Nat Commun. 2017;8:1507.

31. Wright JJ, Konwar KM, Hallam SJ. Microbial ecology of expanding oxygen minimum zones. Nat Rev Microbiol. 2012;10:381-94.

32. Nunoura T, Takaki Y, Hirai M, Shimamura S, Makabe A, Koide O, et al. Hadal biosphere: Insight into the microbial ecosystem in the deepest ocean on Earth. Proc Natl Acad Sci. 2015;112:E1230. 
33. Pachiadaki MG, Yakimov MM, LaCono V, Leadbetter E, Edgcomb V. Unveiling microbial activities along the halocline of Thetis, a deep-sea hypersaline anoxic basin. ISME J. 2014;8:2478-89.

34. Vigneron A, Alsop EB, Cruaud P, Philibert G, King B, Baksmaty L, et al. Comparative metagenomics of hydrocarbon and methane seeps of the Gulf of Mexico. Sci Rep. 2017;7:16015.

35. Sackett JD, Kruger BR, Becraft ED, Jarett JK, Stepanauskas R, Woyke T, et al. Four Draft Single-Cell Genome Sequences of Novel, Nearly Identical Kiritimatiellaeota Strains Isolated from the Continental Deep Subsurface. Microbiol Resour Announc. 2019;8:e01249-18.

36. Toesch M, Schober M, Faber K. Microbial alkyl- and aryl-sulfatases: mechanism, occurrence, screening and stereoselectivities. Appl Microbiol Biotechnol. 2014;98:1485-96.

37. Sunda W, Kieber DJ, Kiene RP, Huntsman S. An antioxidant function for DMSP and DMS in marine algae. Nature. 2002;418:317-20.

38. Kiene RP, Linn LJ, Bruton JA. New and important roles for DMSP in marine microbial communities. J Sea Res. 2000;43:209-24.

39. Durham BP, Sharma S, Luo H, Smith CB, Amin SA, Bender SJ, et al. Cryptic carbon and sulfur cycling between surface ocean plankton. Proc Natl Acad Sci. 2015;112:453.

40. Cruaud P, Vigneron A, Fradette MS, Charrette $S$, Rodriguez M, Dorea $C$, et al. Open the SterivexTM casing: An easy and effective way to improve DNA extraction yields. Limnol Oceanogr Methods. 2017;15:1015-20.

41. Suzuki MT, Taylor LT, DeLong EF. Quantitative analysis of small-subunit rRNA genes in mixed microbial populations via 5'-Nuclease assays. Appl Environ Microbiol. 2000;66:4605-14.

42. Yu Y, Lee C, Kim J, Hwang S. Group-specific primer and probe sets to detect methanogenic communities using quantitative real-time polymerase chain reaction. Biotechnol Bioeng. 2005;89:670-9.

43. Klindworth A, Pruesse E, Schweer T, Peplies J, Quast C, Horn M, et al. Evaluation of general 16S ribosomal RNA gene PCR primers for classical and next-generation sequencing-based diversity studies. Nucleic Acids Res. 2013;41.

44. Teske A, Sorensen KB. Uncultured archaea in deep marine subsurface sediments: have we caught them all? ISME J. 2007;2:3-18.

45. Vigneron A, Alsop EB, Lomans BP, Kyrpides NC, Head IM, Tsesmetzis N. Succession in the petroleum reservoir microbiome through an oil field production lifecycle. ISME J. 2017;11:2141.

46. Schloss PD, Westcott SL, Ryabin T, Hall JR, Hartmann M, Hollister EB, et al. Introducing mothur: Open-source, platform-independent, community-supported software for describing and comparing microbial communities. Appl Environ Microbiol. 2009;75:7537-41.

47. Pruesse E, Quast C, Knittel K, Fuchs BM, Ludwig W, Peplies J, et al. SILVA: a comprehensive online resource for quality checked and aligned ribosomal RNA sequence data compatible with ARB. Nucleic Acids Res. 2007;35:7188-96. 
48. Bolger AM, Lohse M, Usadel B. Trimmomatic: a flexible trimmer for Illumina sequence data. Bioinformatics. 2014;30:2114-20.

49. Magoč T, Salzberg SL. FLASH: fast length adjustment of short reads to improve genome assemblies. Bioinformatics. 2011;27:2957-63.

50. Yuan C, Lei J, Cole J, Sun Y. Reconstructing 16S rRNA genes in metagenomic data. Bioinformatics. 2015;31:i35-43.

51. Bankevich A, Nurk S, Antipov D, Gurevich AA, Dvorkin M, Kulikov AS, et al. SPAdes: A new genome assembly algorithm and its applications to single-cell sequencing. J Comput Biol. 2012;19:455-77.

52. Markowitz VM, Mavromatis K, Ivanova NN, Chen I-MA, Chu K, Kyrpides NC. IMG ER: a system for microbial genome annotation expert review and curation. Bioinformatics. 2009;25:2271-8.

53. Li D, Liu C-M, Luo R, Sadakane K, Lam T-W. MEGAHIT: An ultra-fast single-node solution for large and complex metagenomics assembly via succinct de Bruijn graph. Bioinformatics. 2015;31:1674-6.

54. Kang DD, Froula J, Egan R, Wang Z. MetaBAT, an efficient tool for accurately reconstructing single genomes from complex microbial communities. Rahmann S, editor. PeerJ. 2015;3:e1165.

55. Parks DH, Imelfort M, Skennerton CT, Hugenholtz P, Tyson GW. CheckM: assessing the quality of microbial genomes recovered from isolates, single cells, and metagenomes. Genome Res. 2015;25:1043-55.

56. Kanehisa M, Araki M, Goto S, Hattori M, Hirakawa M, Itoh M, et al. KEGG for linking genomes to life and the environment. Nucleic Acids Res. 2008;36:D480-4.

57. Caspi R, Altman T, Billington R, Dreher K, Foerster H, Fulcher CA, et al. The MetaCyc database of metabolic pathways and enzymes and the BioCyc collection of Pathway/Genome Databases. Nucleic Acids Res. 2014;42:D459-71.

58. Buttigieg PL, Ramette A. A guide to statistical analysis in microbial ecology: a community-focused, living review of multivariate data analyses. FEMS Microbiol Ecol. 2014;90:543-50.

59. Hammer $\varnothing$, Harper D, Ryan P. PAST-palaeontological statistics, ver. 1.89. Palaeontol Electron. 2001;4.

\section{Figures}




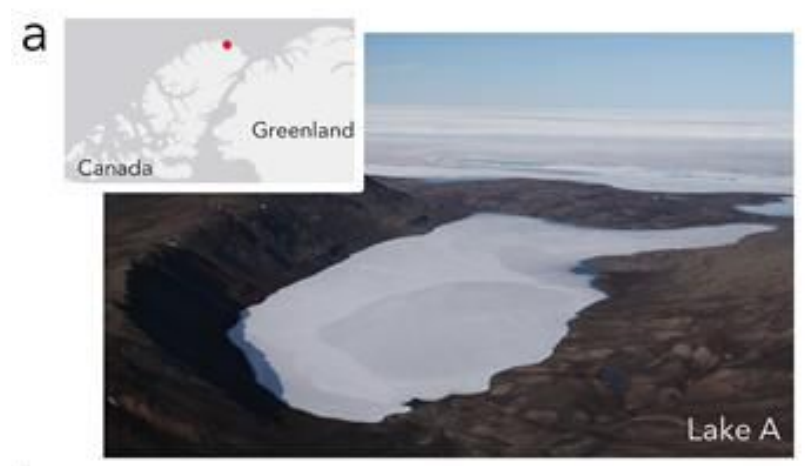

\begin{tabular}{llllllllllllllllll}
\hline & 0 & 10 & 20 & 30 & 40 & 0 & 5 & 10 & 15 & 20 & 25 & 0 & 50 & 100 & 150 & 200
\end{tabular}

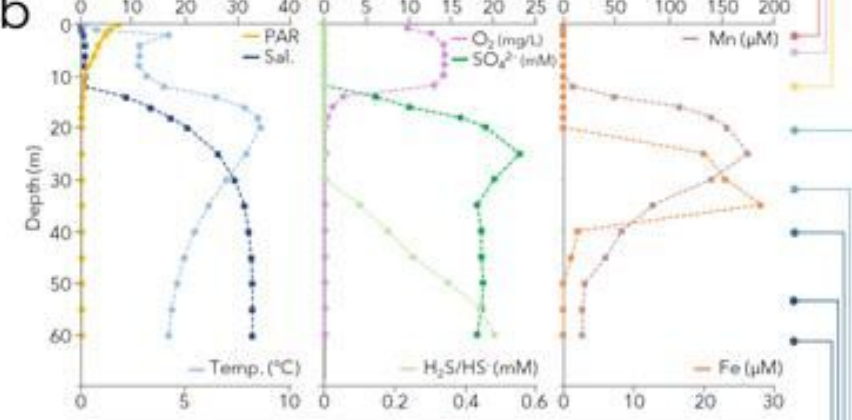

C

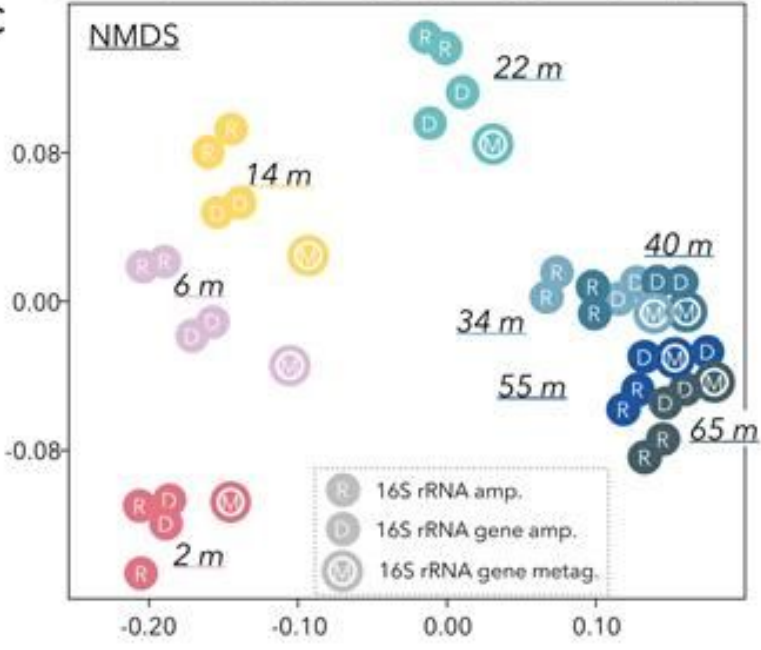

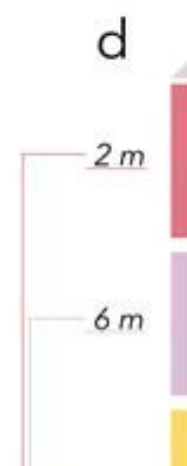

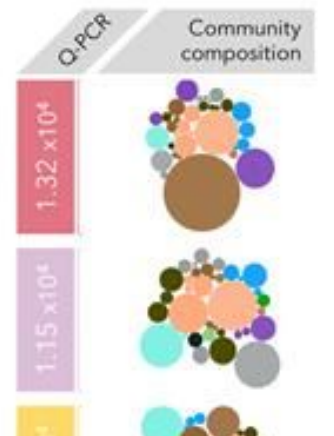

$14 m$

$22 m$
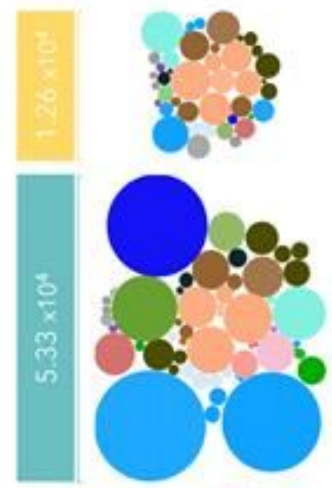

$-34 m$
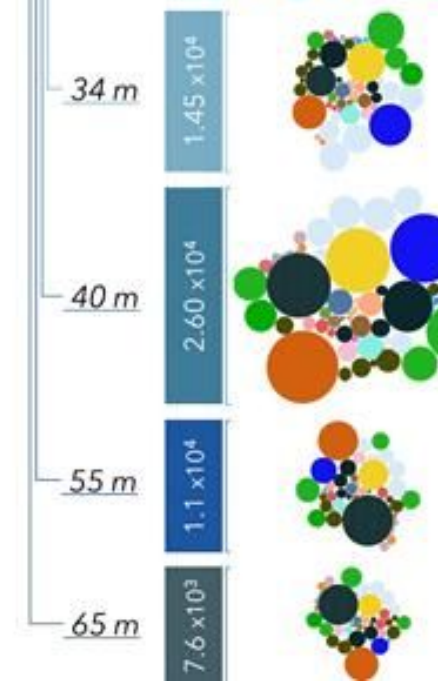

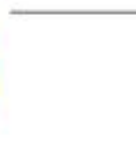

Bray-Curtis dissimilarity
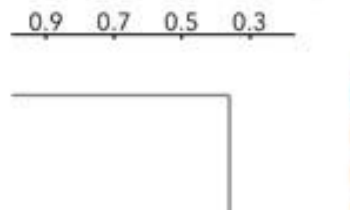

Aminicenantia

Aquiluna

$\mathrm{HgCl}$

Actinobacteria

Atribacteria

Flavobacteriales

Bactercidetes

Chlorcbiaceae

OPB56

Dehalococcoidia

Chiloroflexi

Cyanobacteria

Campylobacteraceae

Kiritimatiellaecta

Latescibacteria

Lentisphaerae

Marinimicrobia

Nitrospinae

Omnitrophicaeota

Patescibacteria

Planctomycetes

Pelagibacter/SAR11

Rhodospirilalles

Alphaproteobacteria

Desulfobacteraceae

Deltaproteobacteria

Gammaprotecbacteria

- Burkholderiaceae

- Betaprotecbacteria

Chthoniobacteraceae

- Verrucomicrobia

- WOR1

Group_Deep_LakeA

\section{Figure 1}

Overview of the physicochemical and microbial features of the Lake A. a) General location and picture of the Lake A. b) Physicochemical profiles of the lake. Photosynthetically active radiation data are derived from Charvet et al. 2012, S042-, H2S, Mn and Fe were extrapolated from Gibson et al. 2002. c) NMDS of the bacterial community based on Bray-Curtis dissimilarity index calculated with $16 \mathrm{~S}$ rRNA amplicons and metagenomic datasets. d) Relative bacterial abundance (qPCR) and community composition based on 16S rRNA genes from metagenomes. The size of the circles represents the relative abundance of genes for each lineage. Only lineages with relative proportions $>1 \%$ of the reads in at least one sample are shown. Overall size of the community is related to qPCR data. qPCR data are expressed in number of 16S rRNA genes per $\mathrm{mL}$ of Lake water. 


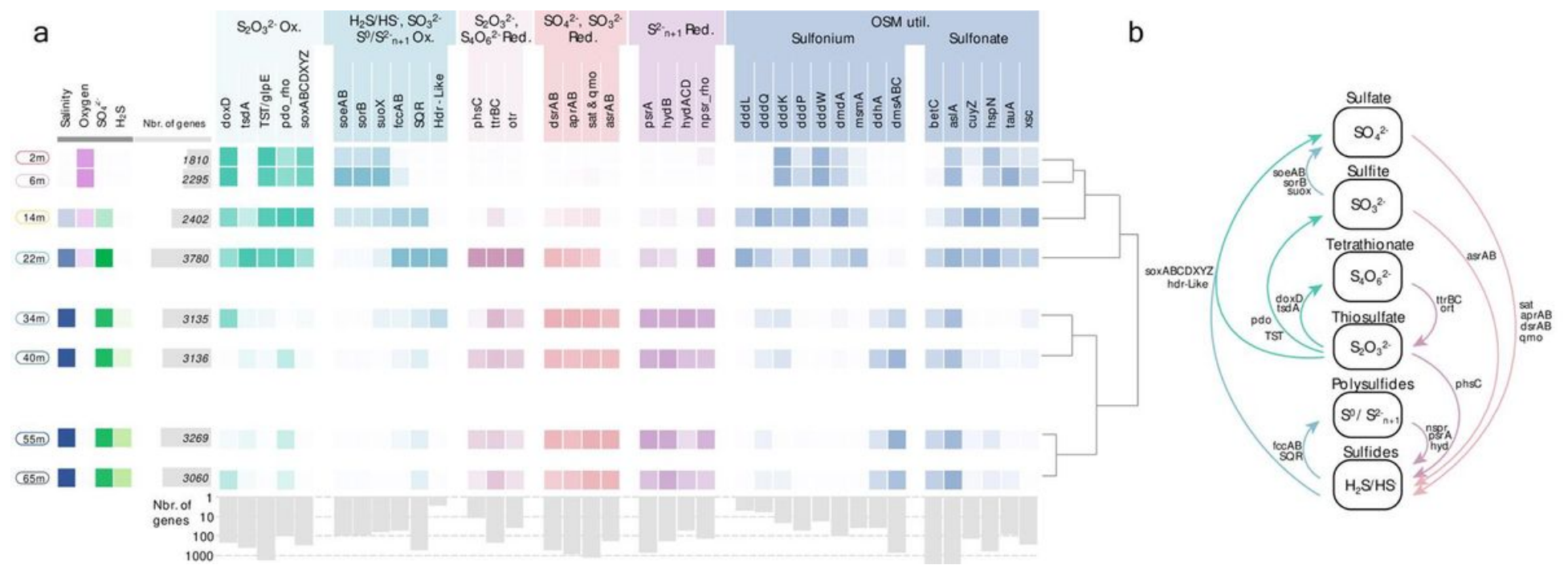

Figure 2

Distribution of sulfur cycling genes. a) Vertical profiles of sulfur cycling genes identified in metagenomes along geochemical gradients (heat map of salinity, oxygen, SO42- and $\mathrm{H} 2 \mathrm{~S}$ ). Hierarchical clustering at the right was computed based on all KEGG orthologues identified in metagenomes. Number of genes per sample and per gene are after normalization. b) Schematic representation of the sulfur cycle 


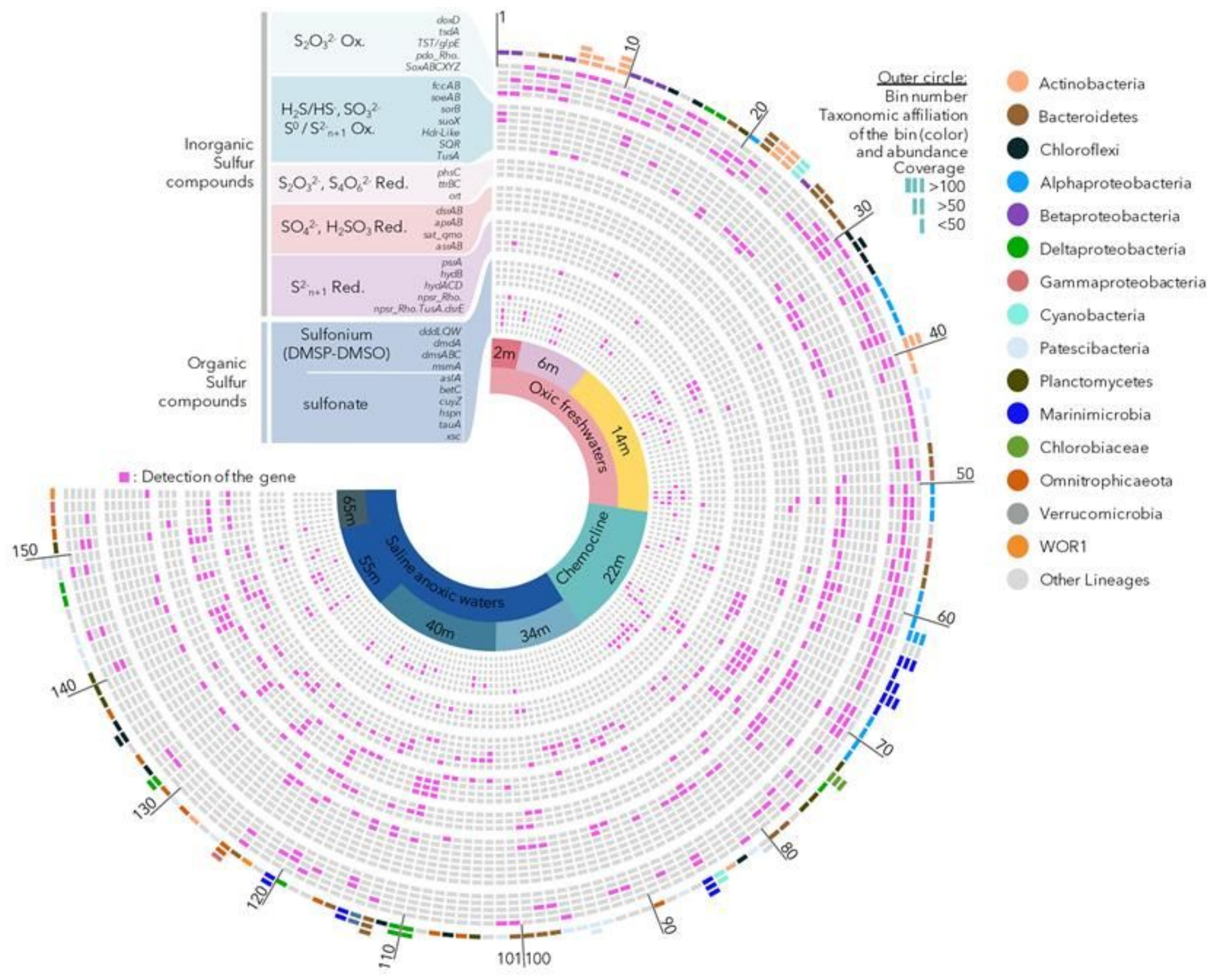

\section{Figure 3}

Key genes for inorganic and organic sulfur transformation identified in the 154 genomic bins with sulfur cycling genes. Colors in the outer circle represent the taxonomic affiliations of the bins. Bins are sorted per depth where they have been recovered. Ox. Oxidation Red. Reduction. Details are provided in supplementary Table S1. 


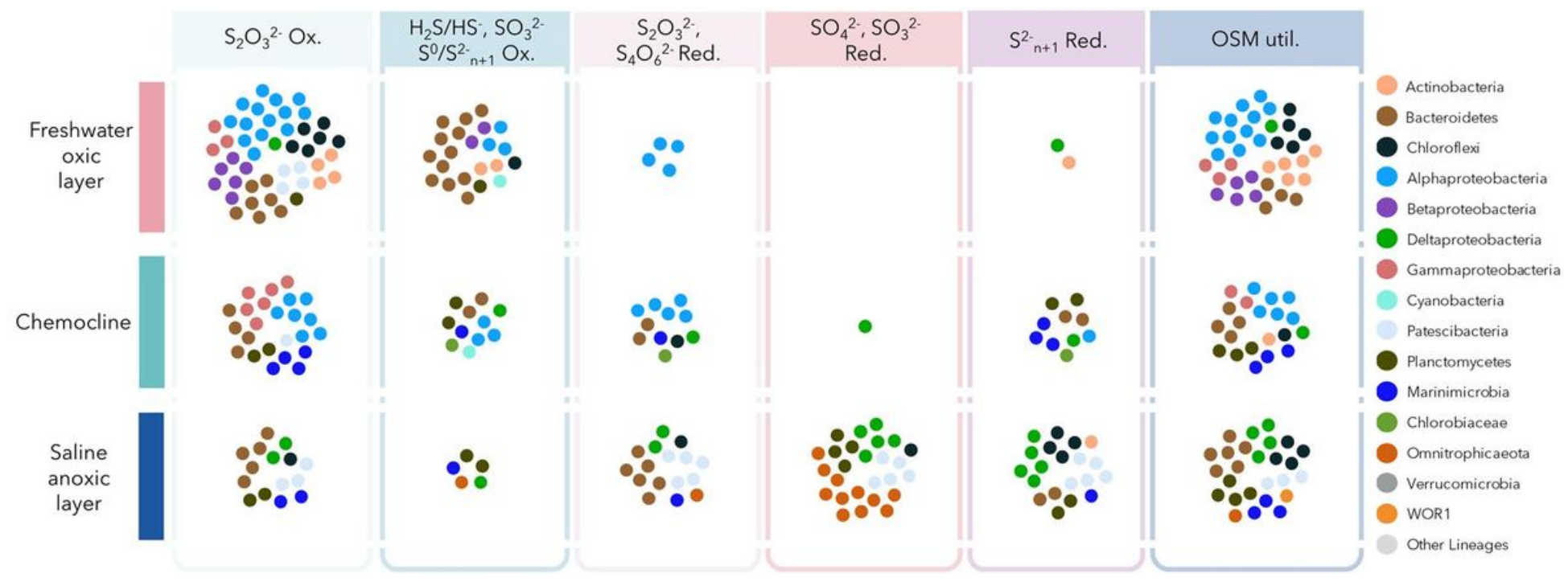

\section{Figure 4}

Depth distribution of metabolic potentials identified in the genomic bins. Each point represents a genomic bin with the color corresponding to its taxonomy. 


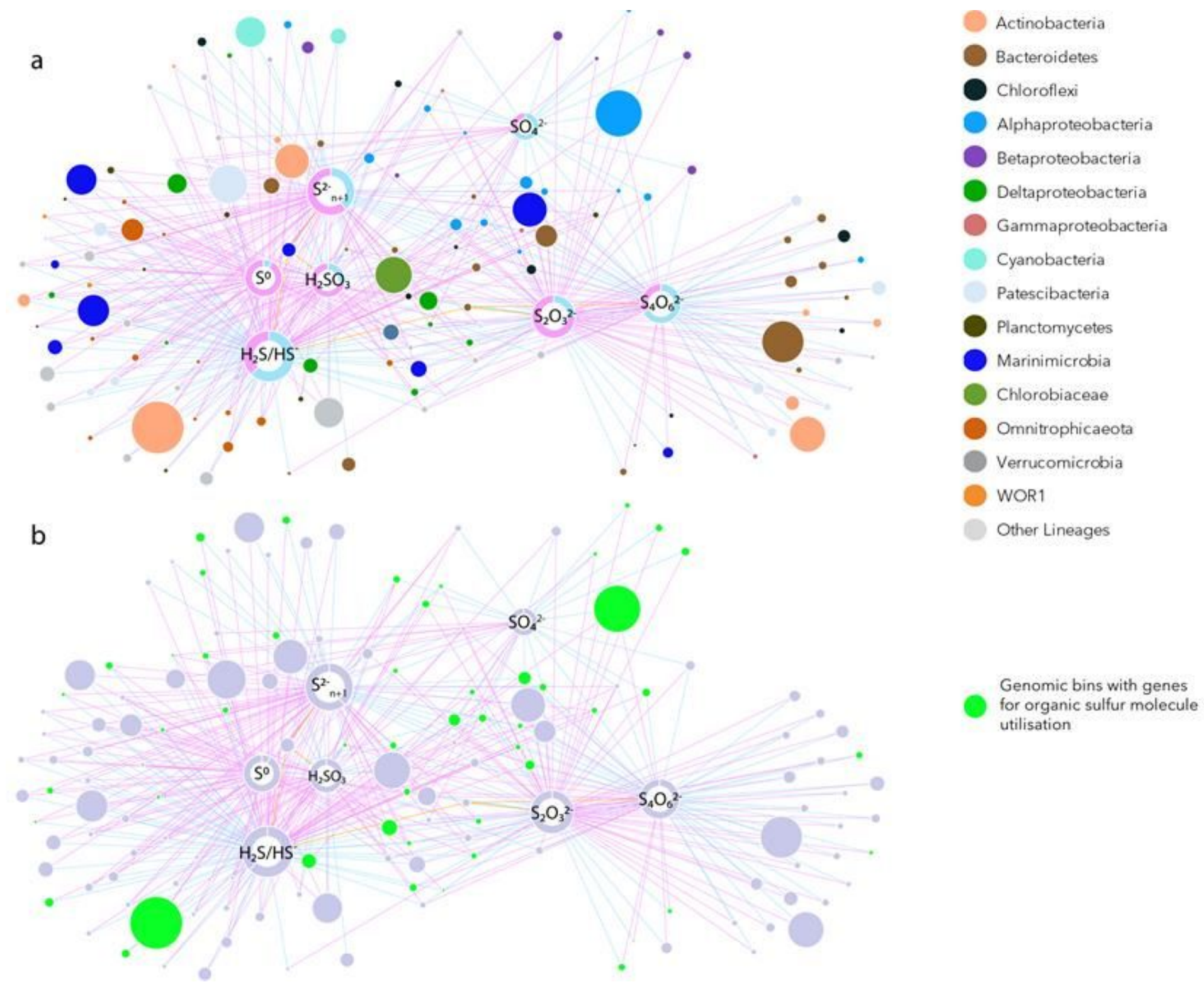

\section{Figure 5}

Metabolic network of the Lake A. . a) Genomic bins are represented as circles with color corresponding to their taxonomic affiliation and size corresponding to the average number of reads mapping on the genomic bin. Genomic bins are connected to sulfur compounds (white circle with pie chart) predicted to be utilized (blue link) or produced (purple link). For each sulfur molecule, pie chart represents the proportion of consumption (blue) and production (purple) and the size of the pie chart is proportional to the number of connections. b) Metabolic network overlaid with the potential for organic sulfur molecule utilisation in green.

\section{Supplementary Files}

This is a list of supplementary files associated with this preprint. Click to download.

- SupplementaryTableS1.xlsx 
- SupplementaryFigureS1.jpg

- SupplementaryFigureS2.jpg

- SupplementaryFigureS1.tif

- SupplementaryFigureS2.tif 